\title{
Sartre lector de Husserl: \\ ¿Es el existencialismo sartreano una hérésie
}

ESTUDIOS ARTÍCULOS DE INVESTIGACIÓN husserliana?

Sartre reader of Husserl: Is Sartrean existentialism a Husserlian hérésie?

\section{Sergio González Araneda'}

Universidad de Santiago de Chile

Recibido 1 agosto 2020 . Aceptado 26 septiembre 2020

\section{Resumen}

La relación filosófica entre Husserl y Sartre es posible rastrearla no solo como maestro-discípulo, sino también como un discípulo que intenta superar a su maestro. El presente artículo es un esfuerzo por mostrar la relación entre la lectura que Sartre hace de Husserl, advirtiendo hasta qué punto el filósofo francés comprende el proyecto del padre de la fenomenología, y el modo en que esta lectura permite a Sartre elaborar un auténtico pensamiento existencialista. Con ello ponemos de relieve la presunta deuda del existencialismo sartreano respecto de la fenomenología husserliana. Palabras claves: Existencialismo, fenomenología, Husserl, Sartre

\section{Abstract}

The philosophical relationship between Husserl and Sartre can be traced back not only as a teacher-disciple, but also as a disciple trying to outdo his teacher. This article is an effort to show the direct relationship between Sartre's reading of Husserl, noting to what extent the French philosopher understands the project of the father of phenomenology, and the way in which this reading allows Sartre to elaborate an authentic thought existentialist. With this we highlight the presumed debt of Sartrean existentialism regarding the Husserlian phenomenology.

Keywords: Existentialism, phenomenology, Husserl, Sartre 


\section{1・Introducción}

En 1953, en un contexto pujante de la fenomenología, Paul Ricoeur afirma que "la fenomenología es en gran medida la historia de las herejías [héré-

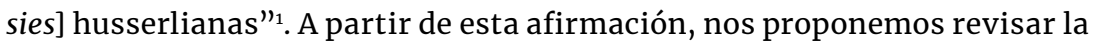
recepción que tiene la filosofía de Husserl en Sartre, proponiéndola como base para su existencialismo fenomenológico ${ }^{2}$. Para ello, es preciso advertir ciertos elementos que determinan la comprensión que tiene el nobel francés respecto del padre de la fenomenología.

Es necesario destacar que el registro de las obras de Husserl en la producción sartreana se limita a sus principales textos publicados en vida: Logische Untersuchungen I y II, Ideen I, Vorlesungen zur Phänomenologie des inneren Zeitbewusstseins, Formale und Transzendentale Logik y Cartesianische Meditationen. A diferencia, por ejemplo, de Merleau-Ponty ${ }^{3}$, Sartre no da señales de haber estudiado los textos inéditos de Husserl, a pesar de que sí hay indicios que muestran su conocimiento sobre la existencia de tales obras. En L'imagination (1936) refiriéndose al problema husserliano de la modificación de neutralidad que emparejaría a la imagen con la percepción, Sartre sostiene: "Tales son las breves alusiones que Husserl hace a una teoría que ha precisado sin duda en sus cursos y en sus obras inéditas, pero que

1 Ricoeur, P. "Sur la phénoménologie" en Esprit 1, 21, 1953, p. 836.

2 La relación filosófica entre Husserl y Sartre ha sido rastreada desde perspectivas exegéticas como desde enfoques críticos-interpretativos. Respecto del primer caso, Stephen Priest ha realizado un notable trabajo entre ambos filósofos en The Subject in Question. Sartre's Critique of Husserl in The Transcendence of the Ego, desplegando un análisis comparativo sobre el lugar del sujeto y los problemas metodológicos del planteamiento husserliano. Para una lectura crítica de la relación entre ambos filósofos, véase la obra de Manfred Frank. Frank se nutre tanto de Husserl como de Sartre, específicamente, se ha inspirado en la tesis del cogito pre-reflexivo sartreano para repensar la intersubjetividad fenomenológicamente dada. Estúdiese su reciente publicación Präreflexives Selbstbewusstsein.

3 Maurice Merleau-Ponty tuvo acceso a textos inéditos de Husserl durante su visita al Archives-Husserl de Louvain en 1939. 
en las Ideen es aún muy fragmentaria" ${ }^{4}$. Sin embargo, no vuelve a pronunciarse al respecto.

Consecuentemente, el conocimiento que Sartre posee del proyecto fenomenológico husserliano es sumamente parcial y limitado. Por ello, revisaremos las principales críticas que Sartre enuncia sobre la fenomenología husserliana, llegando, incluso, a identificar la intencionalidad husserliana con las categorías kantianas del entendimiento.

En este contexto, el presente trabajo está compuesto por cinco partes. En primer lugar, expondremos el contexto histórico-filosófico que sostiene la relación entre ambos filósofos. Luego, revisaremos el conocimiento que Sartre posee de Husserl, pues, como notaremos, se ciñe a la etapa de la fenomenología estática de Husserl, desconociendo el progreso genético alcanzado por el filósofo alemán. En tercer y cuarto lugar, haremos énfasis en la principal distancia que Sartre toma de Husserl: la conciencia pre-reflexiva en el marco de una intencionalidad esencialmente activa, develando la falta de motivo para el método de la reducción fenomenológica y una auténtica ontología del fenómeno. Finalmente, daremos cuenta que el principal descubrimiento sartreano, el cogito pre-reflexivo ${ }^{5}$, es fundamento metodológico para la elaboración de su existencialismo fenomenológico.

\section{2 - Contexto histórico-filosófico entre Husserl y Sartre}

Previo a profundizar en la lectura sartreana de la fenomenología estática de Husserl, es necesario realizar una breve contextualización filosófico-histórica sobre la relación de ambos filósofos. Pretendemos situar la lectura que Sartre hace de Husserl, puesto que la consideramos limitada, al menos en lo que respecta al proyecto de la fenomenología genética en Husserl.

En febrero de 1929, Husserl presentó dos lecciones de fenomenología en La Sorbonne bajo el título de L'introduction a la phénoménologie trascendentale, lecciones que se han intitulado Las conferencias de París. El mismo

4 Sartre, J.-P.: La imaginación. Madrid: Editorial Sarpe, 1984, p. 189.

5 Frank, M.: "Welche Gründe gibts es, Selbstbewusstsein für irreflexiv zu halten", en ProtoSociology 2013, p. 1. 
año, en la École Normale Supérieure, Sartre obtiene el primer lugar del concurso de agrégation en filosofía. No existe certeza sobre la asistencia de Sartre a las lecciones realizadas por Husserl ${ }^{6}$, de lo que sí tenemos certeza, es que Sartre conoce la obra de Husserl gracias a la influencia del libro de Emmanuel Lévinas titulado Théorie de l'intuition dans la phénoménologie de Husserl, así como del libro de Georges Gurvitch Les tendances actuelles de la philosophie allemande, ambos publicados en 1930.

Posteriormente, en 1933, Sartre reside en Alemania como becario en el Institut Français de Berlín. Su estadía resultó determinante para su vida intelectual, pues tuvo la oportunidad de estudiar a fondo la fenomenología trascendental estática de Husserl y, en menor medida, la fenomenología hermenéutica heideggeriana. Desde este momento, el filósofo francés se alinea con la crítica husserliana al psicologismo, llegando a publicar en 1936 L'imagination, y dos años más tarde Esquisse d'une théorie des émotions. Sin embargo, previo a tales publicaciones, en 1934, comienza la redacción de La transcendence de l'Ego, obra clave en el pensamiento fenomenológico, pues, en esta temprana obra, ya se afirma que la conciencia es una absoluta nada (néant) y, por tanto, su sentido se halla instalado en el mundo, como conciencia tética de sí, haciéndose y reconociéndose en el mundo: tesis central en el existencialismo que desarrollará desde la década del cuarenta.

Ahora bien, Sartre no solo estudia detenidamente la fenomenología estática husserliana, sino que realiza una pertinente crítica, con lo cual intenta redefinir ciertos aspectos fundamentales del método fenomenológico. De este modo, toma distancia de la idea husserliana de conciencia, en tanto "conciencia de algo", pues tal noción supondría la existencia de "contenido de conciencia", lo que Sartre necesita rechazar para sostener primacía ontológica de la conciencia pre-reflexiva por sobre la conciencia reflexiva. La conciencia, en tanto nada-de-ser, será la actividad pura del intencionar.

Tal redefinición implica, por un lado, sentar la dualidad de las pre-

6 En la presentación de Las conferencias de París, Zirión Quijano escribe: "Escucharon a Husserl [...] Lévy-Bruhl, Lichtenberger, Andler, X. León, E. Meyerson, Jean Cavaillés, Emmanuel Lévinas, Gabriel Marcel, Eugéne Minkowski, J. Patočka, A. Koyré, J. Herring, Émile Baudin, Émile Goblot, Albert Shweitser, Leon Chestov". Husserl, E. Las conferencias de París. México: Universidad Nacional Autónoma de México, 1988, p. viii. 
misas ontológicas del ser-en-sí y ser-para-sí, que conducen a Sartre hacia lo que denominamos existencialismo fenomenológico; por el otro, suprimir la noción husserliana de yo trascendental que opera como sedimentación histórica de sentido respecto de la conciencia, supeditando la idea del yo, esta vez, a una conciencia-nada que actúa y se compromete en tanto es absolutamente libre de acción.

De este modo, el conocimiento que Sartre tiene de Husserl, y, precisamente, de la fenomenología trascendental, se convierte en un re-conocimiento en cuanto el filósofo francés se plantea redefinir la conceptualización que da forma a la fenomenología. En un breve escrito, pero de gran profundidad filosófica, titulado Une idée fondamentale de la Phénoménologie de Husserl: l>intentionnalité Sartre describe lo que se comprende por conciencia intencional en Husserl, a la vez que plantea, en comparación con el ser-en-el-mundo heideggeriano, lo que comprende él por conciencia intencional:

La filosofía de la trascendencia nos arroja al camino real, entre las amenazas, bajo una luz enceguecedora. [...] Ser es estallar en el mundo, es partir de una nada de mundo y de conciencia para de pronto estallarse-conciencia-en-el-mundo. Si la conciencia trata de recuperarse, de coincidir al fin con ella misma, en caliente, con las ventanas cerradas, se aniquila. A esta necesidad que tiene la conciencia de existir como conciencia de otra cosa que ella misma, Husserl la llama "intencionalidad"?.

Sabemos que Sartre estudió a Husserl con el interés que un discípulo estudia a su maestro. De hecho, el primero de febrero de 1940 escribe a Simone de Beauvoir: "Husserl se había apoderado de mí, veía todo a través de las perspectivas de su filosofía, que además me resultaba más accesible, por su apariencia de cartesianismo. Yo era "husserliano"'s.

Este período husserliano se extiende aproximadamente por cuatro años'. En este periodo, Sartre escribe L'imaginaire, La transcendence de l'ego y comienza, en 1937, la redacción de un gran libro que queda inconcluso, La 
Psyché. Aun así, algunas partes fueron publicadas bajo el título de Esquisse d'une théorie des émotions.

A lo largo de este periodo, Sartre reconoce escribir en contra de Husserl, "pero en la medida en que un discípulo puede escribir contra su maestro" ${ }^{\prime 10}$. De allí en más, Sartre se distancia de la fenomenología husserliana y comienza una dudosa lectura de Heidegger. El principal motivo que distanció a Sartre de Husserl se debe a la concepción de intencionalidad, ya que, a juicio del francés, Husserl no lograría escapar del todo de la filosofía kantiana. Tal como lo revisaremos, esta afirmación de Sartre se muestra en sus textos de la década del treinta y se extiende hasta $L 〉 \hat{E} t r e$ et le Néant. De tal modo, para Sartre la fenomenología trascendental derivaría en un nuevo tipo de idealismo:

Un foso cada vez más profundo me separaba de Husserl: en el fondo su filosofía evolucionaba hacia el idealismo, cosa que yo no podía admitir, y sobre todo, como todo idealismo o como toda doctrina que simpatiza con él, su filosofía tenía su materia pasiva, su "Hylé", a la que una forma viene a moldear (categoría kantiana 0 intencionalidad) ${ }^{11}$.

Como sea, lo cierto es que el conocimiento que posee Sartre sobre la obra de Husserl y la tradición fenomenológica contribuyó a la formación de una fenomenología francesa de corte existencial; de allí la importancia de revisar su influencia. Merleau-Ponty da testimonio de esto cuando, refiriéndose a la formación del espíritu existencial de la filosofía francesa a mediados del siglo $\mathrm{XX}$, sostiene:

Todos los filósofos de los que he hablado hace un momento y que son todos filósofos de la existencia en algún sentido, Sartre los ha conocido, quiero decir, ha conocido sus obras, en particular en el curso de una estadía que hizo en el Institut Français de Berlín en los años que precedieron a la guerra. Me acuerdo bastante bien que a su regreso nos hizo leer a todos a Husserl, Scheler, Heidegger, por ejemplo, que eran ya conocidos 
en Francia pero cuyas obras no estaban plenamente difundidas en aquel momento. Se trataba aquí por tanto de una formación verdaderamente filosófica que él se había dado ${ }^{12}$.

\section{3· Fenomenología husserliana desde Sartre}

El conocimiento que Sartre tiene de la fenomenología de Husserl se limita a su inicial aspecto trascendental-estática, comprendiéndola como aprehensión de esencias individuales dentro de un marco de experiencias posibles. De hecho, Sartre se aventura infundadamente a colocar bajo el título de fenomenista la filosofía de Husserl. En relación a la búsqueda del sentido estructural del para-sí, es decir, la negación, señala Sartre:

Husserl [...] permaneció temerosamente en el plano de la descripción funcional. Por eso no sobrepasó nunca la pura descripción de la apariencia en tanto que tal, se encerró en el cogito, y merece ser llamado, pese a sus protestas, fenomenista más bien que fenomenólogo' ${ }^{13}$.

En este contexto, es necesario señalar los principios de la filosofía de Husserl a los que Sartre se refiere y critica extralimitándose en duros términos.

Al comienzo de su proyecto fenomenológico, Husserl sostiene que la intencionalidad es el núcleo de las relaciones entre la conciencia y el fenómeno. Pero, para sostener la objetividad de los correlatos de conciencia, necesita emprender una crítica radical al ejercicio intencional en razón de superar cualquier inmanentismo que conduzca al psicologismo, naturalismo o historicismo.

Así, la estructura de la intentionalitas es dada mediante la correlación ego cogito cogitatum, es decir, por un lado, se encuentra la vivencia-del-objeto en tanto multiplicidad de los actos reales subjetivos (intentio); por otro, el objeto-de-vivencia en tanto unidad ideal de sentido, unidad como lo 
pensado (intentum). Para esta primera etapa de Husserl, la idealidad de los objetos trasciende la realidad de los actos subjetivos, de allí que "la intencionalidad [sea] lo que caracteriza la conciencia en su pleno sentido y lo que autoriza para designar a la vez la corriente entera de las vivencias como corriente de conciencia y como unidad de una conciencia"14.

Una descripción fundamental de la intencionalidad de la conciencia está presente en la célebre Quinta investigación, cuando Husserl sostiene que "sólo hay presente una cosa, la vivencia intencional, cuyo carácter descriptivo esencial es justamente la intención respectiva. Esta constituye plena y exclusivamente el representar este objeto o el juzgar sobre él, etc."15.

La intencionalidad husserliana, por tanto, es una intencionalidad activa, procedente, en último término, del sujeto o del yo puro. Sergio Rábade habla de "sujeto-conciencia pura que, como tal, es esencialmente abertura al mundo de objetos, sin el que sería ininteligible"16. Desde luego, sobre este punto surge el problema operacional del sujeto al cual se encuentra anclada la conciencia intencional, pues:

Si llevo a cabo la epojé fenomenológica, sucumbo, como el mundo entero en la tesis natural, también "yo, el hombre" a la desconexión, quedando la pura vivencia del acto con su propia esencia. Pero yo veo también que el apercibirla como vivencia humana, prescindiendo de la tesis de existencia, acarrea toda clase de cosas que no necesitan forzosamente estar también ahí, y que, por otra parte, no hay desconexión capaz de borrar la forma del cogito y el sujeto "puro" del acto: el "estar dirigido a" [...] encierra necesariamente en su esencia esto: ser, justo, un "desde el yo", o, en un rayo de dirección inversa, "hacía el yo", y este yo es el puro, al que no puede hacerle nada ninguna reducción ${ }^{17}$.

Efectivamente, la conciencia intencional no solo supone la existencia de un correlato de objetividad esencial en la aprehensión de lo real, 
sino que, a la vez, dota al sujeto empírico de una constitución ideal de orden trascendental que opera como estabilidad objetiva de sentido. García-Baró, en un análisis similar al realizado por Sartre, señala que la primera etapa de Husserl platoniza la fenomenología al defender que, además de fenómenos mentales y no mentales, existen realmente esencias dispuesta al conocimiento del hombre ${ }^{18}$.

A esto, debemos agregar un componente fundamental para nuestros objetivos, y es la idea que existe una diferencia no de grado, sino esencial, entre la materia intencional y los contenidos primarios. Dicho de otro modo, existe una diferencia esencial entre noema, que hace referencia al modo de darse el objeto a la conciencia, y noesis, que apunta al acto mismo de la conciencia de intencionar o referirse al objeto. Es importante poner de relieve este punto, puesto que Sartre se pronuncia sobre la naturaleza y estructura de la conciencia intencional husserliana:

Justamente porque es espontaneidad pura, porque nada puede morder en ella, la conciencia no puede actuar sobre nada. Así, el esse est percipi exigiría que la conciencia, pura espontaneidad que no puede actuar sobre nada, diera el ser a una nada trascendente conservándole su nada de ser: total absurdo. Husserl intentó salvar estas objeciones introduciendo la pasividad en la noesis: es la hyle o flujo puro de lo vivido y materia de las síntesis pasivas [...] La hyle, efectivamente, no podría ser conciencia; si no, se desvanecería en translucidez ${ }^{19}$.

El sentido trascendental de la fenomenología husserliana queda de manifiesto no solo en la elaboración de una nueva concepción de la intencionalidad, sino que, especialmente, se pone de manifiesto al momento de su crítica al psicologismo:

Las ciencias empíricas son ciencias de "hechos". Los actos de conocimiento del experimentar que les sirven de fundamento sientan lo real como individual, lo sientan como existente en el espacio y en el tiempo, como algo que existe en este punto del 


\section{tiempo tiene esta su duración y un contenido de realidad que por esencia hubiera podido existir igualmente bien en cualquier otro punto del tiempo ${ }^{20}$.}

Frente a esta postura que relativiza la constitución esencial de la realidad, Husserl sostiene que "al sentido de todo lo contingente es inherente tener precisamente una esencia y por tanto un eidos que hay que aprehender en su pureza" ${ }^{21}$. En efecto, existe una estabilidad de orden objetivo-universal que subyace a la realidad fenoménica, y que por tanto, es preciso trascender para aprehender su sentido originario. Es decir, se requiere de una suspensión de lo asumido acríticamente, para hallar fenomenológicamente su sentido constituyente.

Husserl, desde la publicación de Logische Untersuchungen, se encarga de profundizar el estudio de la filosofía en los objetos inmanentes de la conciencia intencional. Al dotar de sentido ideal el modo de ser categorial del acto intencional, centra su proyecto filosófico en la intuición categorial, es decir, en la adecuación de los ingredientes reales del acto de pensamiento a una estructura de racionalidad que trasciende al acto mismo.

A lo largo de Logische Untersuchungen II, Husserl articula el esquema que determina el programa de la fenomenología trascendental. En su Sexta investigación, se establece la diferencia entre la estabilidad atemporal e ideal de los objetos de pensamiento y la factualidad temporal de los actos de pensamiento del sujeto. Realizar esta distinción no solo dota a la conciencia de un carácter trascendental, sino que radicaliza la idea de conciencia intencional. Debido a que, por un lado, la esfera noemática, o lo estrictamente pensado, se vuelve trascendente al acto mismo de pensar (lo noético), de forma tal que la idealidad lógico-matemática opera como legalidad en el yo empírico-material. Por otro lado, la esfera de la noesis, o lo relacionado al acto puro de pensar, se dota de temporalidad y espacialidad, es decir, queda definido como ser sensible (reell), y como tal, sujeto a la idealidad trascendental que lo tensiona.

El método husserliano, al llevar a cabo la operación reflexiva de la epojé fenomenológica, no solo produce la puesta entre paréntesis de la va- 
lidez mundanal, sino que sienta las condiciones necesarias para el acceso al ego trascendental. Esto resulta fundamental, pues, como se verá, es uno de los puntos de inflexión entre la filosofía de Husserl y Sartre, dado que, para el segundo, la conciencia, en tanto nada-de-ser, se define por su actividad misma, sin contenido de conciencia, y no por la adecuación a un correlato de sentido pre-dado.

Si bien en Logische Untersuchungen se presenta el mecanismo de la epojé mediante la reducción eidética, su profundización y complejización se encuentra en Ideen con la presentación de la epojé o reducción trascendental, operación mediante la cual es posible acceder a la subjetividad trascendental. Este acceso se presenta como legalidad de sentido en cuanto desconecta la creencia de la realidad mundanal y nos revela la constitución esencial de esta realidad.

La reducción trascendental, desde la perspectiva de Sartre, constata una individualización eidética de la realidad mundanal, de la realidad natural. De ello se sigue que aquella presencia de orden natural adquiere objetividad y sentido solamente cuando es puesta entre paréntesis, cuando es reducida a su pura constitución esencial. Pues, en ese momento, la presencia mundanal, se presenta a la conciencia como objeto fenomenológico, constituyéndose como objeto-de-vivencia.

Así, la reducción trascendental desconecta al sujeto de la mera creencia natural del mundo y lo conduce a una radical significación del mundo en tanto mundo constituido y vivido por él. Como menciona Sartre "la fenomenología [husserliana] es una descripción de las estructuras de la conciencia trascendental fundada en la intuición de las esencias de tales estructuras"22.

\section{Sartre, trascendentalidad e intencionalidad}

A partir de lo señalado, es decir, del entendimiento que tiene Sartre sobre la fenomenología de Husserl, la fenomenología sartreana se distanciará de la fenomenología trascendental-estática en cuanto interpreta que la con- 
ciencia intencional husserliana es constituida mediante una dimensión que esencialmente se distancia de la presencia fenoménica, y con ello, se hallaría en una situación de jerarquía de sentido. Así, la conciencia aparece para Sartre como un absoluto sin interioridad, una conciencia puramente exterior, que se hace conciencia de sí cuando entra en contacto con la presencia del fenómeno.

Con esto, el filósofo francés redefine la estructura de la intencionalidad realizada por Husserl, puesto que la conciencia al ser primariamente pre-reflexión, es decir, una absoluta nada-de-ser, se halla arrojada hacia el mundo, y lo constituye al posicionarse como exterioridad reflexiva de sí. Dicho de otro modo, "la conciencia no se limita a proyectar significaciones afectivas sobre el mundo que le rodea: vive en el nuevo mundo que acaba de crear" 23 .

En este contexto, el principal desacuerdo con Husserl se centra en el recurso del yo trascendental que, tras las reducciones eidética y fenomenológica, se presentaría como conditio sine qua non para la conciencia. Es pues, la reducción fenomenológica -que Sartre ironiza llamándola "operación erudita"- el mecanismo para distinguir al yo trascendental del yo psicológico y otorgarle un principio trascendental de unificación, constitución y significación. Sartre criticará este punto señalando categóricamente que no existe un yo en o detrás de la conciencia, sino más bien un yo para la conciencia: "Todos los resultados de la fenomenología amenazan ruina si el Yo no es, del mismo modo que el mundo, un existente relativo, o sea un objeto para la conciencia" 24.

Negar tanto la reducción eidética, como el yo trascendental husserliano es condición para sostener que la conciencia no posee contenidos, por tanto, no posee esencia ni naturaleza determinista, tesis central para su filosofía: "la fenomenología no necesita recurrir a este Yo unificador e individualizador. En efecto, la conciencia se define por la intencionalidad. Por la intencionalidad, se trasciende a sí misma, se unifica escapando de sí"25.

Cuando Sartre escribe que la intencionalidad es la conciencia tras-

23 Sartre, J.-P. Bosquejo de una teoría de las emociones. Madrid: Alianza Editorial, 2015, p. 86.

24 Sartre, J.-P. La trascendencia del ego. Madrid: Editorial Síntesis, 2003, p. 42.

25 Ibid., p. 38. 
cendiéndose a sí misma, está definiendo su método fenomenológico: la nihilización (néantisation). Allí la conciencia emerge sin contenido ni esencia alguna, pues su objeto, por naturaleza, está fuera de ella, es decir, emerge como pura espontaneidad. Por esta vía, Sartre resulta ser severamente crítico con la fenomenología husserliana:

[El] Yo superfluo es nocivo. Si existiera, arrancaría a la conciencia de sí misma, la dividiría; se deslizaría en cada conciencia como una lámina opaca. El Yo trascendental es la muerte de la conciencia. [...] [S]i el Yo es una estructura necesaria de la conciencia, este Yo opaco queda elevado de inmediato al rango de absoluto. Nos vemos ahora en presencia de una mónada. Tal es, por desgracia, la orientación del pensamiento nuevo de Husserl [refiriendo a Cartesianische Meditationen] ${ }^{26}$.

Y sobre el contenido hylético del vivenciar de la conciencia que anima la intencionalidad, y que es indicativa de un yo trascendental ${ }^{27}$, Sartre, en su ensayo sobre ontología fenomenológica, señalará:

\section{Husserl, a lo largo de toda su carrera filosófica, estuvo} obsesionado por la idea de la trascendencia. Pero los instrumentos filosóficos de que disponía, en particular su concepción idealista de la existencia, le privaban de los medios de dar razón de esa trascendencia; su intencionalidad no es sino la caricatura de ella ${ }^{28}$.

En efecto, desde la lectura sartreana, para Husserl toda conciencia es intencional en la medida que aprehende esencialmente lo real; por tanto posee una estructura trascendental que es necesaria para otorgar sentido a la realidad aprehendida. Esta estructura, que opera como unificación de los actos de conciencia y constitución del significado de los correlatos inten-

26 Ibid., p. 39-41.

27 Desde este análisis se vislumbra la cuestionable objeción de solipsista dirigida a Husserl. Sobre esto, estúdiese el texto de Pedro Alves "Empatía y ser-para-otro. Husserl y Sartre ante el problema de la intersubjetividad". Allí, en un notable giro, Alves presenta al cogito de Sartre como solipsista y al de Husserl como auténtica fenomenología de la comunicación.

28 Sartre, J.-P. El ser y la nada, p. 193. 
cionales, es accesible solo mediante la suspensión de sentido del yo psíquico. Evidentemente la lectura que realiza Sartre de la fenomenología husserliana es, por lo menos, problemática. Especialmente sobre este último punto, donde, al parecer, Sartre limita el mecanismo fenomenológico husserliano como una operación platonizada, donde habría una existencia ideal-esencial sobre un mundo espurio. A este respecto, es necesario recordar las palabras de Ricoeur en su introducción a la versión francesa de Ideen I, donde despeja el sentido real de la reducción fenomenológica:

Esta es la razón de que la intencionalidad pueda ser descrita
antes y después de la reducción fenomenológica; antes, ella es
un encuentro; después, es una constitución. Ella sigue siendo
el tema común de la psicología pre-fenomenológica y de la
fenomenología trascendental. La reducción es el primer gesto
libre, porque es liberador de la ilusión mundana. A través de él
yo pierdo en apariencia el mundo que verdaderamente gano ${ }^{29}$.

\section{5 - Sobre la ontología del fenómeno}

Ahora bien, al rechazar la reducción reflexiva de Husserl, Sartre propone una néantisation activa desde la conciencia pre-reflexiva. En este sentido, la conciencia es intencionalidad en su espontaneidad que tiende a revelar sentidos y valores de la realidad, creándola. La conciencia, por tanto, es la condición existencial de creación y transformación del mundo a partir de una nada constituyente de sentido.

Para la fenomenología sartreana la expresión "conciencia es conciencia de algo" significará, por tanto, el movimiento de trascendencia de la conciencia a lo que no es ella misma: es su apertura fundamental a lo otro dado en su fenómeno mismo. En este sentido, la conciencia es superación de sí misma, puesto que ella se presenta como nada absoluta que encuentra profundidad de ser solo cuando se trasciende. Así, el concepto de relación intencional sartreano permite descubrir la realidad no solo desde una exigencia epistemológica, sino también desde un plano ontológico y afectivo, 
siendo este cambio de enfoque fundamental.

La filosofía francesa [...] no conoce ya apenas más que la epistemología. Pero para Husserl y los fenomenólogos, la conciencia que adquirimos de las cosas no se limita a su conocimiento. El conocimiento o pura "representación" no es sino una de las formas posibles de mi conciencia "de" este árbol; puedo también amarlo, temerlo y odiarlo, y ese excederse de la conciencia a sí misma, a la que se llama "intencionalidad", se vuelve a encontrar en el temor, el odio y el amor ${ }^{30}$.

Podemos notar que Sartre interpreta la idea de intencionalidad desde una perspectiva prioritariamente ontológica, frente a la preeminencia epistemológica que encontraríamos en Husserl:

La conciencia es conciencia de algo: esto significa que la trascendencia es estructura constitutiva de la conciencia; es decir, que la conciencia nace conducida sobre un ser que no es ella misma. Es lo que llamamos la prueba ontológica. Se responderá, sin duda, que la exigencia de la conciencia no demuestra que esta exigencia deba satisfacerse. Pero esta objeción no puede mantenerse frente a un análisis de lo que Husserl llama intencionalidad, y cuyo carácter esencial ha desconocido. Decir que la conciencia es conciencia de algo significa que para la conciencia no hay ser ${ }^{31}$.

Con todo, no es cierto que Sartre reduzca la intencionalidad a una exclusiva salida hacia lo otro-exterior. En L'imagination (1936) Sartre diferencia entre la imagen y la percepción según la correlación noético-noemático, a partir de la estructura de las síntesis intencionales ${ }^{32}$. Por otro lado, a lo largo de L'imaginaire (1940) presenta la intencionalidad ya no como salida a un exterior trascendente, sino que, esta vez, la intencionalidad opera como la posibilidad de negación del mundo mediante el cual la conciencia 
puede desplegarse imaginariamente. Este poder de negación, que se fundamenta en la tesis de irrealidad del noema, es, para Sartre, la "otra cara de la libertad" ${ }_{33}$. Desde este lugar adquiere radical importancia la relación entre intencionalidad e imaginario en la arquitectura sartreana.

En este contexto, resulta interesante que una de las primeras críticas que Sartre realiza a Husserl se relaciona con la conciencia de imagen. Para Sartre, Husserl no habría dado solución a la actividad de la conciencia de producir imágenes, pues la limita a una materia hylética subjetiva que la intención anima ${ }^{34}$. Esto supone que la conciencia intencional se despliega sobre un suelo pasivo y subjetivo que Sartre identificará con la arquitectura kantiana de las categorías del entendimiento: "Aun cuando se concediera a Husserl que hay en la noesis un estrato hilético, no sería concebible cómo la conciencia puede trascender esta subjetividad hacia la objetividad"35. De este modo, la teoría de Husserl no resulta "sensiblemente diferente de la de Kant. Pues, en efecto, si mi Ego empírico no es más seguro que el del prójimo, Husserl ha conservado el sujeto trascendental, radicalmente distinto de aquél y harto parecido al sujeto kantiano" ${ }^{36}$.

Según sus escritos, Sartre no estudió el curso de 1904-1905, actualmente conocido como Husserliana XXIII, pues allí Husserl profundiza en su teoría acerca de la conciencia de imagen y phantasie en relación con la corriente temporal de la conciencia, lo que supone una superación del presunto vacío en el que habría caído la explicación de Husserl.

Ahora bien, sobre la fenomenalidad del fenómeno, en Conscience de soi et connaissance de soi Sartre sostiene que "el examen de la conciencia no tética revela un cierto tipo de ser que denominaremos existencia [pues] el existente es lo que no es y no es lo que es"37. Veámoslo nuevamente. La conciencia se funda y constituye como absoluta nada, no entendida como un vacío absurdo del cual es imposible desprender rendimiento epistemológico o estético, sino que, por el contrario, es una nada que se-crea en tanto in-

33 Sartre, J.-P. Lo imaginario. Buenos Aires: Editorial Losada, 2005, p. 256.

34 Sartre, J.-P. La imaginación, p. 185.

35 Sartre, J.-P. El ser y la nada, p. 27.

36 Ibid., p. 306.

37 Sartre, J.-P. "Conciencia de sí y conocimiento de sí" en Acta fenomenológica latinoamericana, 5, 2016, p. 348. 
tenciona su existencia, es decir, se arroja sobre lo que es, diremos con Sartre, ser-en-sí. Dicho de otra manera, se produce el surgimiento de la conciencia y su constitución esencial (o intencional) en dialéctica relación con aquellas existencias que coinciden plenamente con su ser: "El ser en-sí no es jamás ni posible ni imposible: simplemente es" ${ }^{\prime 38}$.

Este arrojo nihilizante es lo que constituye la existencia, pues la dinámica del arrojo representa una escisión que se extiende a un nivel tanto ontológico como epistemológico. Esto se debe a que ilustra el salto constituyente de la nada al ser, de la pre-reflexión a la reflexión, en definitiva, es el paso de una conciencia no tética a una conciencia tética.

Este punto nos remite a una pregunta medular en la filosofía sartreana ¿puede decirse que el fenómeno tiene un ser, como se dice que tiene un color, una forma, una gravedad? Sartre responde afirmando que "la aparición es el conjunto de los fenómenos que se presentan al conocimiento para ser enlazados. Pero este aparecer tiene en sí mismo un ser. No se trata de un ser que remite a un sustrato misterioso, sino que en tanto que aparición, es"39.

El ser del aparecer es lo que otorga significado a la condición existencial, en tanto es forma y contenido del cogito reflexivo. Sin embargo, esta conciencia reflexiva, que Sartre denomina "formación segunda", es el fin epistemológico de una condición ontológica previa, a saber, la del cogito pre-reflexivo que, en tanto absoluto nada-de-ser, es constituyente de la relación transfenoménica fundada entre el ser del fenómeno y el fenómeno del ser:

Una descripción de la conciencia pre-reflexiva no revelará un objeto-otro, porque quien dice "objeto"; dice probable. El otro o bien debe ser cierto, o bien debe desaparecer. Lo que descubriremos en el fondo de nosotros mismos, es al otro, no como conocimiento particular, ni como factor constitutivo del conocimiento del conocimiento en general, sino en tanto que "concierne" a nuestro ser concreta y ónticamente en las circunstancias empíricas de nuestra facticidad ${ }^{40}$. 


\section{$6 \cdot$ Cogito pre-reflexivo como premisa del existencialismo fenomenológico}

Nos hemos propuesto mostrar hasta qué punto el existencialismo fenomenológico sartreano se forma como una herejía husserliana. Ahora podemos dar completa muestra de ello, pues la filosofía sartreana surge en tanto apunta sus críticas al método de la reducción y a la conceptualización que está contenida en ella. En una carta fechada el 20 de enero de 1940 Sartre escribe a Simone de Beauvoir:

Lo que hacíamos hasta ahora, como pequeños y aplicados fenomenólogos, era una ontología. Buscábamos las esencias de la conciencia con Husserl o el ser de los existentes con Heidegger. Pero la metafísica es una 'óntica'. Ahora ponemos las manos en la masa, ya no consideramos las esencias [...] sino directamente las existencias concretas y dadas, y nos preguntamos por qué son así las cosas ${ }^{41}$.

La crítica de la fenomenología husserliana -y ahora también la heideggeriana- apunta a todo aspecto esencialista que determine y posibilite la existencia en y del mundo. Sartre en sus estudios fenomenológicos sobre la imaginación y la emoción descubre la posibilidad humana de distanciarse del objeto dado inmediatamente como real. Con esto, abre la posibilidad de situarse "fuera de lo real", de aquello presente como escenario absoluto y determinado. De este modo, la libertad fundamental de la conciencia se expresa en la realización de aquello no-presente, de aquello imaginario.

A partir de este marco conceptual, Sartre describe la función del cogito pre-reflexivo, o conciencia irrefleja, como condición ontológica de la absoluta espontaneidad con la que emerge la conciencia. Dicho de otro modo, la pre-reflexión no solo permite una reflexión sobre el mundo, sino que es la posibilidad de transformarlo, pues, el cogito pre-reflexivo tiene la estructura de la conciencia (de) sí, esto es, una conciencia no tética de sí como siendo conciencia de algo que ella no es.

$4 \mathbf{1}$ Sartre, J.-P. Cartas al castor y a algunos otros. Buenos Aires: Editorial Sudamericana, 1987, p. 37. 
En su Esquisse d'une théorie des émotions Sartre muestra la operación afectiva de la pre-reflexión y la manera en que se constituye la operación reflexiva (operación de segundo grado). Por ejemplo, es evidente que cualquier persona, en cualquier situación puede cobrar consciencia de su estado emocional, afirmar que está furioso o que siente miedo. Sin embargo, "el miedo no es originalmente conciencia de tener miedo, como tampoco la percepción de este libro es conciencia de percibir el libro"42. Esto se debe a que la conciencia es ante todo irreflexiva y, por tanto, solo puede ser conciencia de sí en un modo no posicional. En efecto, el individuo que tiene miedo, tiene miedo de algo, el sujeto que está furioso, lo está respecto de algo que le causa furia, es decir, la emoción es un modo determinado de aprehender la realidad, pues "el sujeto emocionado y el objeto emocionante se hallan unidos en una síntesis indisoluble"43.

Así, en el momento que se toma conciencia del miedo que nos constituye, la vivencia del miedo se "congela", de tal manera que la emoción no es la toma de conciencia de aquel sentimiento particular, en este caso el miedo. A lo que apunta este análisis es que cuando se asume una posición reflexiva se paraliza la corriente vital del vivir como se vivencia naturalmente una emoción.

Efectivamente, para Sartre el fenómeno de la emoción representa una transformación del mundo, pero no porque este fenómeno posea una característica única y particular que lo dota de este poder transformador, sino que la característica por la cual es posible re-significar el mundo es precisamente la de constituirse en el plano de la pre-reflexión. Es allí donde la conciencia no guarda nada, donde se expresa como torrente creativo y re-creativo. Recuérdese al personaje más reconocido de la literatura sartreana, Antoine Roquentin, quien nos recuerda que "hacer algo es crear existencia"44.

Ahora bien, cuando Sartre se refiere a la conciencia no tética, pone de manifiesto que la conciencia pre-reflexiva responde a la máxima: la existencia precede a la esencia ${ }^{45}$. Puesto que no hay ningún tipo de condicio-

42 Sartre, J.-P. Bosquejo de una teoría de las emociones, p. 62.

43 Ibid., p. 63.

44 Sartre, J.-P. La náusea. Buenos Aires: Editorial Losada, 2003, p. 262.

45 Sartre, J.-P. El existencialismo es un humanismo. Barcelona: Edhasa, 2004, p. 27. 
namiento, ni naturaleza, ni esencia que determine la conciencia existente, que, justamente por ser un vacío absoluto, es su propia fuente y condición de posibilidad.

Por lo tanto, podemos hablar de dos momentos de la conciencia: (a) un momento original y originario que corresponde al cogito pre-reflexivo, $y$ (b) un cogito derivado, que es el momento en que la conciencia se pone a sí misma como objeto de conocimiento, es decir, se re-flexiona téticamente. Sartre tiene la necesidad de sostener el primer cogito originario, pues en él se lleva a cabo la referencia al objeto. Es decir, corresponde al momento de la intencionalidad irrefleja que actúa desprendida de cualquier interés, revelando una dimensión pre-cognoscitiva que manifiesta la radicalidad del ser-otro para la conciencia: el en-sí.

La estructura compleja de la conciencia es esta: hay un acto irreflejo de reflexión, sin Yo, que se dirige a una conciencia reflejada. Esta se vuelve objeto de la conciencia reflexionante sin cesar, empero, de afirmar su objeto propio [...]. Al mismo tiempo, aparece un objeto nuevo, que da ocasión para una afirmación de la conciencia reflexiva, y que, por tanto, no está ni en el mismo plano que la conciencia irrefleja [...], ni en el mismo plano que el objeto de la conciencia irrefleja ${ }^{46}$.

Como toda conciencia es conciencia de algo, la existencia de un ser transfenomenal debe ser entendida como la intencionalidad pura del cogito pre-reflexivo hacia la existencia del en-sí; luego, en la constitución del cogito reflexivo, la referencia de la conciencia es su propio anclaje en el mundo como conciencia reflejada.

Efectivamente, la conciencia pre-reflexiva es el ideal abstracto de la libertad absoluta de los individuos, pues muestra la operación de una conciencia que no tiene determinación alguna. Es decir, en su sentido más original, la conciencia es pura transparencia, es la Nada (néant). Desde este lugar, Sartre pone énfasis en que la libertad es concretamente el actuar en el mundo, transformarlo en virtud de una articulación subjetiva que tiene como primer develamiento la absoluta pre-reflexión. 


\section{7· Reflexiones finales}

Si observamos con detención, podemos identificar tres argumentos fundamentales por los cuales Sartre, mientras se distancia de la fenomenología husserliana, queda necesariamente determinado por esta. El primero de ellos dice relación con el suelo trascendental que sostendría al pensamiento husserliano. A juicio de Sartre, Husserl recurre a un orden jerárquico-esencial de sentido, del cual la realidad participa y a la cual podemos acceder una vez sea suspendida, reducida o puesta entre paréntesis.

Esta idea se repite incluso en su obra póstuma Cahiers pour une morale cuando afirma que Husserl, al igual que Kant, opera trascendentalmente en el sentido en que la conciencia se despliega según operaciones infinitas que, de algún modo, disuelven el objeto o fenómeno en la conciencia ${ }^{47}$. Por esta línea, el asimilar a Husserl con Kant, le permite a Sartre criticar el recurso del método fenomenológico en tanto devela una articulación esencialista de lo real, que, por tanto, conduciría a una moral contemplativa, en lugar de defender una existencia activa que constituya mundo:

El ideal no es contemplar lo que se hace, sino vivir. Cualquier moralidad que mutile la vida es sospechosa. Así como la epojé fenomenológica según Husserl no elimina un matiz del mundo, del mismo modo la epojé moral no debe quitarle un matiz a la vida humana ${ }^{48}$.

Con esto, encontramos la segunda escisión entre ambos filósofos. Si en Husserl la fenomenología consiste en "reducir" las cosas a su esencia, urge preguntarnos la necesidad de llevar a cabo el método fenomenológico: la reducción eidético-trascendental. Es evidente que Sartre no profundiza en el motor que anima la reducción husserliana, la fenomenología genética. No obstante, para nuestros objetivos, basta con señalar su crítica hacia la falta de motivo para llevar a cabo la reducción. Esto se debe a que si la realidad no comporta un orden de sentido supeditado a una estructura trascendental, entonces la aprehensión esencial de lo real se produce según la 
presencia efectiva del fenómeno, constituido mediante la dinámica de la fenomenalidad del fenómeno. Esto es, el hecho que el fenómeno es absoluto indicativo de sí mismo.

Con esto se abre la tercera crítica. Sartre coloca como fundamento de su teoría la premisa del cogito pre-reflexivo, y, de este modo, puede elaborar el argumento central de su existencialismo fenomenológico: la existencia como absoluto e indicativa de la completa libertad de ser, hacer y reconocer en un mundo intersubjetivo, que, por tanto, implica la responsabilidad de actuar. Quizás donde mejor se expresa esta tesis es en La Nausée: "Desearía tanto abandonarme, olvidarme, dormir. Pero no puedo, me sofoco: la existencia me penetra por todas partes, por los ojos, por la nariz, por la boca"49.

Como se puede notar, la presencia e influencia de la fenomenología de Husserl en los principios y fundamentos del pensamiento sartreano es completamente determinante. Pero solo a la manera de un momentáneo discípulo que, en un momento determinado, tuvo la capacidad de comprender a su maestro y con ello intentar pensar auténtica y autónomamente.

De allí que la deuda filosófica que tiene Sartre con Husserl entra en la categoría de hérésies husserliennes en tanto toma del maestro una base teórica para criticarla (hemos visto que solo parcial y en algunos momentos infundadamente). Con ello propone una nueva perspectiva acerca de la conciencia, el método fenomenológico y la intencionalidad. Esta vez, en clave de existencialismo fenomenológico, donde el dato inmediato que es aprehendido por la conciencia es la complejidad de la existencia en tanto libertad que se hace, acciona y responsabiliza.

Plantear el existencialismo sartreano como una herejía husserliana evidencia que posee premisas y un método estrictamente fenomenológico. Este es el principal objetivo que ha buscado la presente investigación: analizar y evidenciar el latente suelo fenomenológico que posee el existencialismo sartreano. Despejar este suelo, nos permite un nuevo horizonte de análisis respecto del existencialismo de Sartre, esta vez, tomando distancia de interpretaciones que han emparejado y determinado su filosofía a influencias como la de Kierkegaard o la de Heidegger ${ }^{50}$, para, esta vez, acercar

49 Sartre, J.-P. La náusea, pp. 102-103.

50 En L'Unique chez Kierkegaard et Sartre, la destacada profesora Marivonne Perrot planteó la relación entre Kierkegaard y Sartre como una continuación filosófica centrada en 
su arquitectura teórica al padre de la fenomenología, Edmund Husserl.

\section{Bibliografía}

Alves, P. "Empatía y ser-para-otro. Husserl y Sartre ante el problema de la intersubjetividad" en Investigaciones Fenomenológicas 9, 2012, pp. 1138.

De la Puente, C. "La afectividad en la teoría intersubjetividad del joven Sartre" en Areté, 2, 18, 2006, pp. 253-266.

Farrell, N. The New Sartre. Londres: Continuum, 2003.

Frank, M. Präreflexives Selbstbewusstsein. Leipzig: Philipp Reclam, 2015.

Frank, M. [en línea] "Welche Gründe gibts es, Selbstbewusstsein für irreflexiv zu halten", en ProtoSociology (2013), pp. 1-21, www.protosociology.de/on-philosophy.html [Consultado: 20/09/2020].

García-Baró, M. Edmund Husserl (1859-1938). Madrid: Ediciones del Orto, 1997.

Husserl, E. Investigaciones Lógicas. Tomo I y II. Madrid: Alianza Editorial, 1999.

Husserl, E. Las conferencias de París. México: Universidad Nacional Autónoma de México, 1988

Husserl, E. Ideas I. México: Fondo de Cultura Económica, 1962.

el problema ontológico de lo uno y la subjetividad. Si bien compartimos con Perrot en que Kierkegaard es una presencia cercana al desarrollo filosófico de Sartre, creemos inoportuno limitar la génesis del problema sobre la subjetividad y la presencia del otro en su filosofía a la influencia del danés. En este punto, nos parece que Sartre ha hecho uso de los principales descubrimientos husserlianos respecto del espacio intersubjetivo, tal como ha observado agudamente el profesor Carlos de la Puente: "Se puede entender mejor esta cosificación que sufro a través de la mirada del otro si se le analiza desde dos enfoques teóricos. Desde el esquema espacial de Husserl, del que Sartre se apropia, y desde el punto de vista de la intencionalidad de la persona que es mirada" De la Puente, C.: "La afectividad en la teoría intersubjetividad del joven Sartre" en Areté, 2, 18, 2006, p. 259.

Por otra parte, Nik Farrell, en The New Sartre, mostrando la recepción e influencia de Sartre en las derivas posestructuralistas y posmodernas, ha insistido en que la génesis de la filosofía sartreana ha sufrido una influencia heideggeriana determinante. 
Merleau-Ponty, M. "La Philosophie de l'existence" en Dialogue 5, 3, 1966, pp. 307-322.

Perrot, M. "L'Unique chez Kierkegaard" en Pešić, B.: Existence and the One. Buenos Aires: Editorial Teseo, 2019, pp. 147-162.

Priest, S. The Subject in Question. Sartre's Critique of Husserl in The Transcendence of the Ego. Londres: Routledge, 2000.

Rábade, S. Estructura del conocer humano. Madrid: G. Del Toro-Editor, 1969.

Ricoeur, P. "Sur la phénoménologie" en Esprit 1, 21, 1953, pp. 821-839.

Ricoeur, P. "Introducción a 'Ideas I' de Edmund Husserl" en Idées directrices pour une phénoménologie. París: Gallimard, 1950.

Sartre, J.-P. "Conciencia de sí y conocimiento de sí" en Acta fenomenológica latinoamericana, 5, 2016, pp. 343-371.

Sartre, J.-P. Bosquejo de una teoría de las emociones. Madrid: Alianza Editorial, 2015.

Sartre, J.-P. Lo imaginario. Buenos Aires: Editorial Losada, 2005.

Sartre, J.-P. El existencialismo es un humanismo. Barcelona: Edhasa, 2004.

Sartre, J.-P. La trascendencia del ego. Madrid: Editorial Síntesis, 2003.

Sartre, J.-P. La náusea. Buenos Aires: Editorial Losada, 2003.

Sartre, J.-P. El ser y la nada. Buenos Aires: Editorial Losada, 1993

Sartre, J.-P. Cartas al castor y a algunos otros. Buenos Aires: Editorial Sudamericana, 1987.

Sartre, J.-P. La imaginación. Madrid: Editorial Sarpe, 1984.

Sartre, J.-P. Diarios de guerra. Buenos Aires: Editorial Losada, 1983.

Sartre, J.-P. Cahiers pour une morale. París: Gallimard, 1983.

Sartre, J.-P. El hombre y las cosas. Buenos Aires: Editorial Losada, 1960. 